\title{
Dynamics of small features in the solar chromosphere
}

\author{
N. $\mathrm{Al}^{1}$, C. Bendlin ${ }^{2}$, and F. Kneer ${ }^{2}$ \\ 1 Istanbul Üniversitesi, Üniversite Rasathanesi, 34452 Üniversite-Istanbul, Turkey \\ 2 Universitäts-Sternwarte, Geismarlandstr. 11, 37083 Göttingen, Germany
}

Received 16 May 2001 / Accepted 27 November 2001

\begin{abstract}
A spatially highly resolved time series of a quiet region at disk centre taken in the $\mathrm{Na}_{2}$ line with the "Göttingen" spectrometer in the Vacuum Tower Telescope/Tenerife was used to study the dynamic behaviour of different chromospheric features. Two CCDs were employed for simultaneous exposures of narrow-band and "white-light" images forming 128 repetitive "scans" of 30 images each in nearly two hours. The data set yielded line profiles over the two-dimensional field of view which served to create various maps indicating, e.g., vertical velocities or minimum line intensities. The analysis of the features of interest was further based on maps displaying the horizontal velocity field derived from white-light images. The occurrence of various bright points was monitored, showing quite different properties. Some persistent intra-network bright points follow the horizontal flows of the underlying photosphere. During the time series, a "K grain" is seen to occur several times at the same location. The power spectrum of the fluctuation of the minimum intensity of its corresponding $\mathrm{Na}_{2}$ mean line profile exhibits a pronounced absolute maximum for a period of about $24 \mathrm{~min}$. The behaviour of several peculiar intra-network bright points still lacks an unequivocal interpretation. Possibly, the signature of gravity waves has been detected. Further investigation revealed that the power spectra of the fluctuation of Doppler shift and minimum intensity calculated for one of these bright points both show an absolute maximum for a period of $4.3 \mathrm{~min}$. In the course of these fluctuations, time spans of regular oscillations were noticed for all such points lasting from about 15 to $25 \mathrm{~min}$. Power spectra calculated only over these times exhibit pronounced absolute maxima in a rather narrow frequency range.
\end{abstract}

Key words. Sun: chromosphere - Sun: oscillations

\section{Introduction}

The solar chromosphere has been the subject of intensive studies for many decades. The work done so far yielded progress in many fields of research, e.g. the fine structure and dynamics of the chromosphere, the properties and propagation of waves, the mechanism(s) of chromospheric heating, the role of the magnetic field and its connection with certain phenomena occurring on different scales, and their possible interaction with the underlying photosphere (see e.g. Bray \& Loughhead 1974; Rutten \& Uitenbroek 1991; Narain \& Ulmschneider 1996; Kneer \& von Uexküll 1999; and references therein). Yet, it will take further efforts to fully understand the many puzzling findings still left, to detect new items - especially the highly dynamic, small-scale features which are difficult to observe -, and to reveal their overall relation.

For a thorough investigation of the properties of peculiar small features in the solar chromosphere, the employment of a variety of tools is indispensable. The data

Send offprint requests to: F. Kneer, e-mail: kneer@uni-sw.gwdg.de set comprising the two-dimensional time series obtained in the $\mathrm{Na}_{2}$ line with high spatial resolution provides the means for the proper study of many different aspects of such features. The use of the maps and images gained after the data reduction and of power spectra calculated for selected areas in the field of view showing unusual features is presented in the next sections. Maps based on white-light images add supplementary information about the solar photosphere to the properties of the features of interest in the lower to middle chromosphere known from the maps derived from the narrow-band filtergrams. Thus, a comprehensive view of different types of small bright chromospheric features is given here, adding to earlier reports on this many-sided group of phenomena.

\section{Outline of the observations}

The data set underlying this investigation was used before to study chromospheric oscillations (Al et al. 1998a, 1999). As an extensive description of the observations as well as of the data reduction was given in previous publications (Al 1997; Al et al. 1998a; Bendlin \& Volkmer 1995), both topics need not be treated at length here. 
The data set was obtained with the two-dimensional spectrometer based on a universal birefringent filter (UBF) and a Fabry-Perot interferometer (FPI) mounted in the Vacuum Tower Telescope (VTT) at the Observatorio del Teide/Tenerife (Bendlin et al. 1992; Bendlin \& Volkmer 1995). On May 25, 1995, a time series of a quiet region at disk centre was recorded, mainly consisting of 128 pairs of white-light and of narrow-band scans taken strictly simultaneously with two CCDs. Each scan comprises 30 pairs of images. For the narrow-band filtergrams, the FPI was tuned to 30 wavelength settings differing by $\sim 30 \mathrm{~m} \AA$. These images were taken with a spectral resolution of about 200000 in a wavelength range of $\sim 900 \mathrm{~m} \AA$ around the line core of $\mathrm{Na}_{2}$. This procedure was repeated with a regular interval of $56 \mathrm{~s}$ between the start of successive scans, while 30 images were recorded in less than $10 \mathrm{~s}$. With a pixel resolution of $00^{\prime \prime} 2$ and a size of the CCD-chips of 384 pixels $\times 286$ pixels, the field of view covered $76^{\prime \prime} .8 \times 57^{\prime \prime} .2$. The images were taken with an exposure time of $60 \mathrm{~ms}$ and a resolution of 12 bits. Further images needed for the proper reduction of the time series completed the data set (see next section).

\section{Data reduction}

\subsection{Fundamentals}

Any necessary steps were taken to ensure that for the white-light images and their narrow-band counterparts obtained simultaneously the field of view was exactly the same. Distortions induced by the differing light paths leading to CCD 1 and CCD 2 employed to take white-light and narrow-band images, respectively, were removed using a grid whose geometrical pattern was reconstructed via a destretching algorithm. All images were corrected for the dark signal and underwent a suitable flat-field division. Cross-correlation was used to compensate for image motion and seeing-induced distortions. A so-called optimum filter working in the Fourier domain served to reduce the inevitable noise associated with all measurements in both spatial dimensions of the images and in the spectral dimension of the narrow-band filtergrams too. The images with the highest rms value of the intensity determined the "best" or "reference" image of every white-light scan. For each narrow-band scan, the data reduction yielded a line profile for every pixel within the remaining field of view still covering a size of $71^{\prime \prime} 4 \times 52$ " 4 . These profiles were corrected for the blueshift occurring for any position in the field of view off the optical axis due to the fact that the FPI was placed at the image of the telescope's entrance pupil. After the noise reduction (see above), the profiles were used to create velocity maps calculated from their Doppler shifts (via polynomial fits) and minimumintensity images giving the lowest intensity of every profile in the field of view (see Fig. 2). An averaged minimumintensity image was produced in order to discriminate between intra-network and network areas within the field of view as only peculiar small bright features occurring in the intra-network were selected for further investigation. Additionally, some power spectra were computed (see Sect. 4), whose frequency resolution is about $0.14 \mathrm{mHz}$ for the entire time series. The corresponding values for shorter time spans are given in the caption of Fig. 9.

\subsection{Advanced stages}

The narrow-band images belonging to any one scan offer a rare look into different chromospheric heights, which can hardly be provided by other observing techniques. Yet, when looking for features of interest that might fall into the selected category of specimens one has to be careful not to misinterpret these views. Figure 1 shows 16 CCD 2 images (out of 30 making up a complete scan) around the line core of $\mathrm{NaD}_{2}$. Row by row, the wavelength decreases by about $30 \mathrm{~m} \AA$ (from the left to the right). The "line-core image" corresponding closest to the wavelength of the line minimum of a suitably averaged profile is the first one in the third row. Here, the chromospheric network is roughly discernible.

The images in Fig. 1 were scaled individually as to give the impression of about the same average intensity and thus to facilitate the tracing of single structures from image to image through the solar atmosphere.

The line-core image represents the highest layer reaching the lower to middle chromosphere. The other images taken in both wings of $\mathrm{Na}_{2}$ show lower and lower atmospheric heights as they approach (but do not reach) the red or blue continuum, respectively. When trying to interpret the narrow-band images, it is most essential to keep in mind that intensity and velocity information is still mixed here. Yet, the one can be separated from the other resulting in velocity maps and (e.g.) minimum-intensity images. Both help to reveal the nature of several bright points appearing, for example, in the narrow-band scan represented by Fig. 1. The striking chain of four bright dots in the lower left part of the field of view (showing a slight upward slope) which is best seen in the second to fourth image in the third row is just due to high downward velocities as can be deduced from the velocity map in the lower right panel of Fig. 2 showing a chain of dark dots at the same location in the field of view. For the small bright features framed by white rectangles this explanation fails, however, as they appear bright in the red and blue wing. Probably they mark the "bright moustache" phenomenon which is not only observed near sunspots but in network regions too (see e.g. Stellmacher \& Wiehr 1991). As is to be expected in this case, the locations of the suspected moustaches actually correspond to the borders of the supergranular cell seen in Fig. 3. Thus, like any other network phenomenon, they are not considered in Sect. 4 .

The four panels of Fig. 2 show different images belonging to the narrow-band scan of which 16 filtergrams are displayed in Fig. 1. For each of the 128 pairs of whitelight and narrow-band scans, an analogous set of images was obtained and analyzed to gain essential information. 


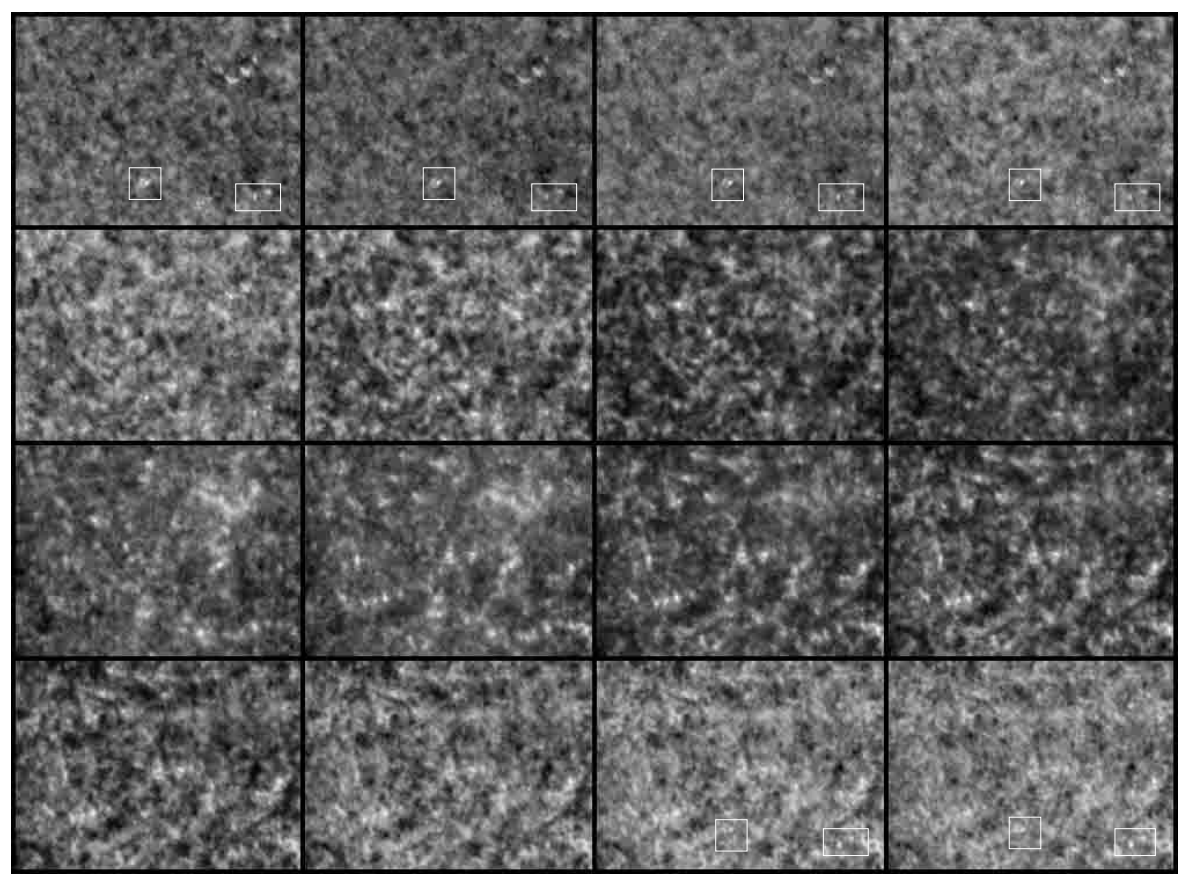

Fig. 1. Narrow-band images $(\Delta \lambda \approx$ $30 \mathrm{~m} \AA$ ) around the line core of $\mathrm{NaD}_{2}$ separated by about $30 \mathrm{~m} \AA$ in wavelength, decreasing row by row from the left to the right; the image corresponding closest to the minimum of the mean line profile ("line-core image") is the first one in the third row; the white squares and rectangles apparently frame "bright moustaches"; field of view: $755^{\prime \prime} 2 \times 55^{\prime \prime} 6$.

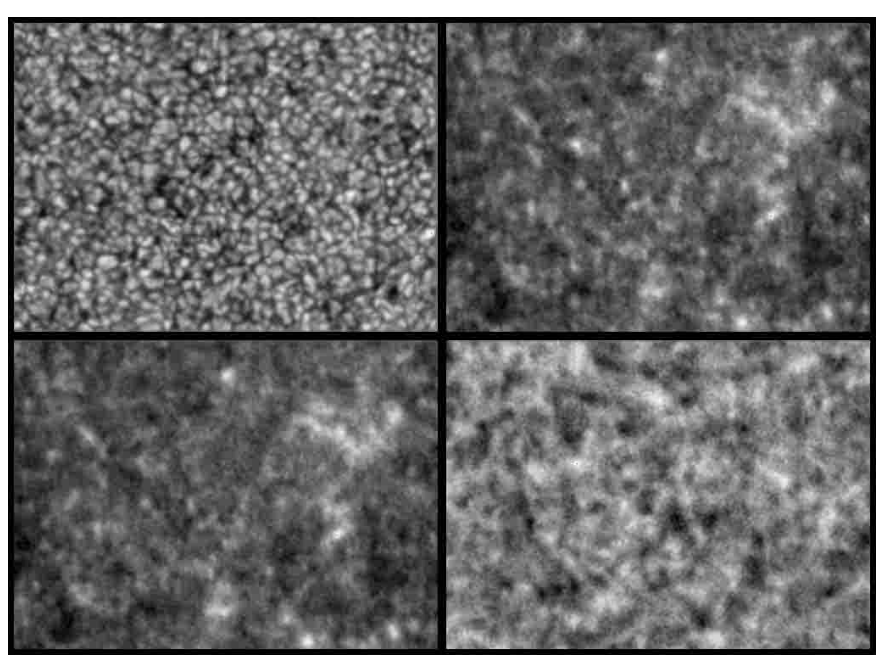

Fig. 2. Upper left panel: "best" (reference) white-light image, upper right: line-core image, lower left: minimum-intensity image, lower right: velocity map (darker/lighter shades of grey represent downward/upward velocities); field of view: $71^{\prime \prime} 4 \times 52^{\prime \prime} .4$.

The white-light image with the highest rms value was declared the best image (cf. upper left panel of Fig. 2). This image was used for comparison whenever features of special interest were found in other images or maps belonging to the same scan to find the corresponding position in the photosphere. The upper right image is the line-core image taken from Fig. 1, and the corresponding minimumintensity image is placed in the lower left panel. Finally, the velocity map reflects the Doppler shifts of the line profiles in the field of view. Darker and lighter shades of grey represent downward and upward flows, respectively. The average velocity is taken to be zero, corresponding to a medium shade of grey.

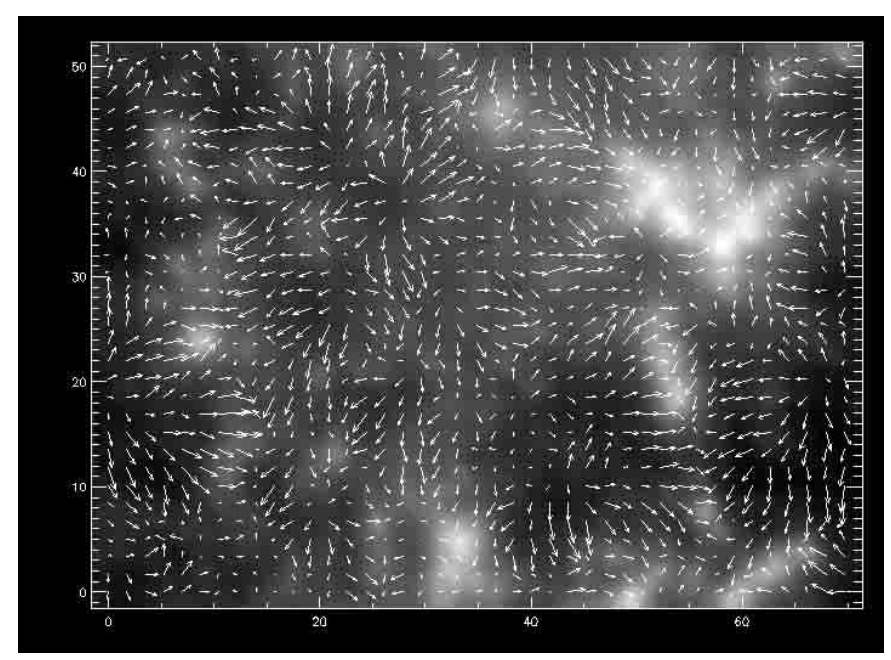

Fig. 3. Horizontal velocity vectors (arrows) superimposed on the averaged minimum-intensity image; their maximum length corresponds to about $1.3 \mathrm{kms}^{-1}$; the spatial axes are labelled in arcseconds.

A good impression of the chromospheric network is given by the averaged minimum-intensity image presented in the background of Fig. 3. Here, only long-lived structures appear bright, thus marking network regions which border a supergranular cell of a typical diameter of about $40^{\prime \prime}$.

The study of the dynamics of intra-network bright features would remain incomplete if it were only based on Doppler shifts. Therefore, some more maps were created which were used to gain information on the horizontal velocity field and the apparent flow pattern at the locations of the bright points under investigation. The arrows in Fig. 3 indicating horizontal velocities were obtained by so-called "local correlation tracking" 
(November \& Simon 1988; Yi 1992; Molowny-Horas 1994) using granules, as seen in the 128 best white-light images, as tracers. Obviously, the dominating horizontal flows on sub-supergranular scales are directed towards the network.

A so-called cork map (not shown here), calculated over an arbitrary length of time, made the small-scale flows within the network even better visible.

Finally, the sources and sinks of the velocity field indicated in Fig. 3 were calculated from the horizontal divergence.

As the maps mentioned above were derived from whitelight images, they provide information about lower heights in the solar atmosphere than the narrow-band filtergrams and the maps calculated from them. Thus, the investigation of chromospheric features can be extended to the conditions existing in the underlying photosphere.

\section{Results}

\subsection{Persistent brightenings}

Figure 4 shows a small area of $15^{\prime \prime} 6 \times 15^{\prime \prime} 6$ in 12 successive minimum-intensity images. As explained in Sect. 2, the temporal distance between neighbouring (minimumintensity) images corresponds to about $56 \mathrm{~s}$. Time proceeds row by row from the left to the right. Here, a pointlike feature appears bright near the centre of the selected field of view. Approximately one hour before, a compact brightening (presumably an ordinary intra-network bright point) already emerged once at the same location. The successive appearance of the persistent bright feature seen in Fig. 4 ends after about $11 \mathrm{~min}$ and thus lasts much longer than the bright phases of $\mathrm{K}$ grains oscillating in intensity.

Another long-appearing chromospheric feature was first observed by Brandt et al. (1992) and named "persistent flasher" owing to its recurrent flashes of brightness. Brandt et al. (1994) studied the migration of these features and found them to move towards the network. The persistent bright feature seen in Fig. 4 also migrates towards the network, taking the same direction as indicated by the flow map for its position. Thus, this bright feature seems to float in the photosphere, as it follows the horizontal flow pattern derived from the white-light images. In Fig. 4, the bright point's migration in the direction of the upper left corner of the images can clearly be seen against the white cross-hairs. Its horizontal velocity reached $1.37 \mathrm{~km} \mathrm{~s}^{-1}$.

Contrary to persistent flashers, the bright feature observed here does not show any oscillation of brightness. Its shape develops from a rather diffuse appearance towards a compact roundish bright area and then dissolves after about $11 \mathrm{~min}$.

In another small intra-network area, a bright point exhibiting similar characteristics was found. During the time of its appearance, it also moved towards the network border (with a velocity of $0.85 \mathrm{~km} \mathrm{~s}^{-1}$ ), in agreement with the direction indicated for this position in the flow map supporting the idea of these bright points being "tied"

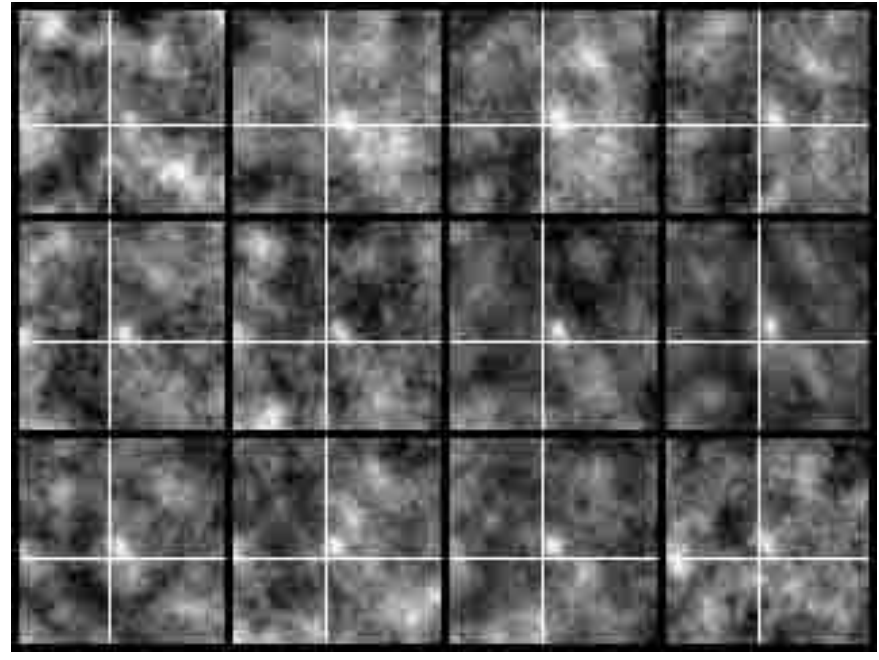

Fig. 4. Small area $\left(15^{\prime \prime} 6 \times 15^{\prime \prime} 6\right)$ in 12 successive minimumintensity images showing a bright point with cross-hairs giving evidence of its migration.

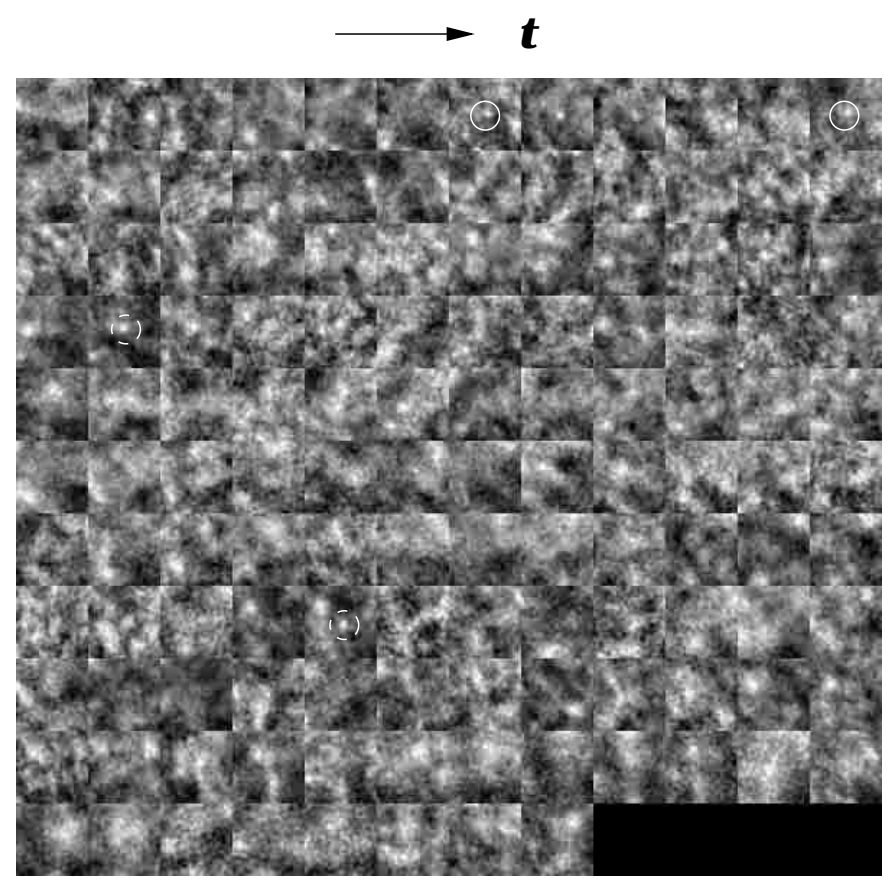

Fig. 5. Temporal development of a selected intra-network area of $10^{\prime \prime} \times 10^{\prime \prime}$ taken from the minimum-intensity images; time $(t)$ proceeds row by row from the left to the right, as indicated by the arrow.

to the photosphere. Because of their longevity and the instance that they follow the convective flows of the photosphere, one may assume that these brightenings are of magnetic origin. This hypothesis is supported by observational evidence of magnetic elements found in several authors' magnetograms moving towards the network for which flow maps and cork maps have been created as well (Title et al. 1992; Wang et al. 1996). Moreover, Zhang et al. (1998) gave a range of lifetimes of intra-network magnetic elements covering the values found here. Further support is given by Nindos \& Zirin (1998). According to 
their work, the velocity of magnetic intra-network Ca II Kline bright features is of the order of $1 \mathrm{~km} \mathrm{~s}^{-1}$ while the non-magnetic elements move much faster (with typical velocities of $35-40 \mathrm{~km} \mathrm{~s}^{-1}$ ).

Interestingly, Cauzzi et al. (2000) state that in analogy with the $\mathrm{Ca}$ II $\mathrm{K}$ line, the $\mathrm{Na}_{2}$ line-centre emission can be used as a proxy for magnetic structures. At least for values of magnetic flux density smaller than a few hundred $\mathrm{G}$ its use is equivalent to that of the Ca II $\mathrm{K}$ emission.

\section{2. $K$ grains}

After Beckers (1964), small-scale bright points in the interior of the chromospheric network conspicuous for maxima of intensity oscillations with large amplitudes in $\mathrm{Ca} \mathrm{K}_{2 \mathrm{~V}}$ are called $\mathrm{K}$ grains. The intensity maxima typically recur 2 to 4 times with periods of about 150 to $240 \mathrm{~s}$ (for a more complete overview see e.g. Rutten \& Uitenbroek 1991 or von Uexküll \& Kneer 1995 and references therein).

In the data set presented here, one bright point was noticed which is possibly related to the dynamic phenomenon producing $\mathrm{K}$ grains in the middle chromosphere. The small intra-network area where it emerged is reproduced in Fig. 5 for the entire time series. The seventh image of the first row shows a bright point clearly contrasted with its surroundings. Its first appearance is marked by a solid white circle, but it is still conspicuous in the subsequent two images. For the next two minutes it remains invisible, but it makes a single appearance in the last image of the first row, marked again by a white circle. No other bright point emerges at the same position until the second image of the fourth row. This reappearance after about $25 \mathrm{~min}$ is marked by a dashed circle. The next period of invisibility lasts nearly $48 \mathrm{~min}$. The final return at the position in question in this time series is marked by another dashed white circle in the fifth image of the eighth row. This behaviour confirms that $\mathrm{K}$ grains may reappear at their former position in the chromosphere as has occasionally been observed for $\mathrm{K}$ grains actually seen in Ca II K $\mathrm{K}_{2 \mathrm{~V}}$ (see e.g. Wellstein et al. 1998 and Damé 1985).

Nevertheless, one should keep in mind that if bright points like the one described above have not been observed in $\mathrm{CaK}$ but in the $\mathrm{NaD}_{2}$ line, their identification with $\mathrm{K}$ grains cannot be proved. It is remarkable that the probability to encounter $\mathrm{K}$ grains in an equally large intranetwork area over the same period is higher when observed in the Ca K line (von Uexküll \& Kneer 1995; Steffens et al. 1996). Yet short lifetimes of the order of one or two minutes and the tendency to reoccur once or several times with an oscillation period of only few minutes is so typical of them that there is no other plausible interpretation to the nature of this bright point at hand. Although it will run under the category of "K grains" in the following, this reservation still applies.

The fluctuation of the minimum intensity $(I)$ of the averaged profile over a small area covering the $\mathrm{K}$ grain $\left(\left(1^{\prime \prime}\right)^{2}\right)$ seen in the upper diagram of Fig. 6 gives the impression of some regular long-period oscillation, confirmed by the power spectrum below showing a pronounced peak at about $0.7 \mathrm{mHz}$ corresponding to $24 \mathrm{~min}$. The second highest peak in Fig. 6 and the "centre" of the dominating double peak in the power spectrum of the Doppler shift (see Fig. 7) belong to about $3.5 \mathrm{mHz}$, while the 3-min oscillations represented by a frequency range from $\sim 5$ to $6 \mathrm{mHz}$ only contribute a minor peak in both power spectra, contrary to other reports. The power spectra in Figs. 6, 7, and also in 8 were smoothed (over 5 neighbouring frequency points) to increase their statistical significance (see Doyle et al. 1999). The pronounced oscillation period of the extraordinary length of $24 \mathrm{~min}$ still awaits an explanation. The averaged power spectrum for the whole intra-network area in the entire field of view gives a dominating period of about $5 \mathrm{~min}$, for comparison. Cauzzi et al. (2000) did not investigate "K grains" seen in $\mathrm{NaD}_{2}$, but they also produced an averaged power spectrum for a larger intra-network area based on data taken in the $\mathrm{NaD}_{2}$ line, yielding a dominating frequency of about $3.5 \mathrm{mHz}$.

Horizontal migration of $\mathrm{K}$ grains as observed by Steffens et al. (1996) and Wellstein et al. (1998) could not be noticed here. These data sets are not too suitable, either, to trace K grains moving as the time interval between successive minimum-intensity images corresponds to the elapsed time between the start of successive scans which is about the lifetime of a $\mathrm{K}$ grain. Thus, $\mathrm{K}$ grains are seen here in only one to maximally three sequential images.

The question of a correlation between the presence of magnetic features and the occurrence of CaII $\mathrm{K}_{2 \mathrm{~V}}$ and $\mathrm{H}_{2 \mathrm{~V}}$ grains in quiet intra-network areas was addressed in a study by Lites et al. (1999). While they concluded that the occurrence of these grains does not depend on the presence of magnetic fields, Sivaraman et al. (2000) found a correlation as high as $85 \%$ between bright points and co-located magnetic elements from pairs of temporally simultaneous CaII K-line spectroheliograms and magnetic area scans.

\subsection{Intra-network bright points in single line-core images}

The detection of four distinct bright points at different locations in single line-core images led to an unsuspected discovery unveiled below. At first glance, they appeared rather inconspicuous as they did not contrast at all with their surroundings in the minimum-intensity images, and their fluctuating brightness did not exceed the average intensity of the intra-network. Therefore, they cannot be considered bright points in the common sense of the term. In order to further study the circumstances under which such phenomena occur, small intra-network areas of $\left(1^{\prime \prime}\right)^{2}$, corresponding to the regions exhibiting a bright point in one line-core image, were scrutinized in different maps and images over large parts of the time series. Figure 8 

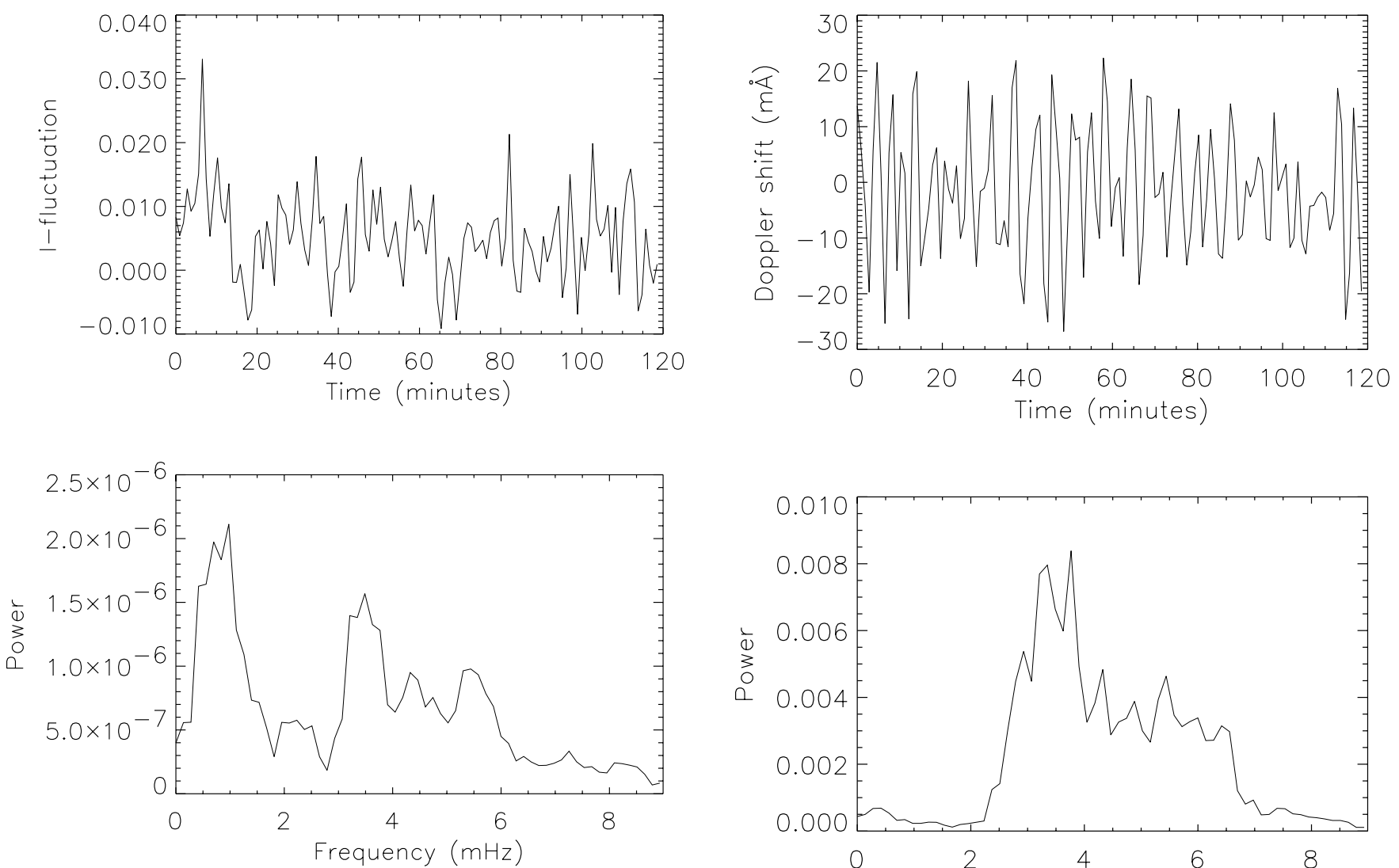

Fig. 6. The upper panel in this figure shows the fluctuation of the line-minimum intensity with time for the position of the bright point seen in Fig. 5; the lower panel gives the corresponding (smoothed) power spectrum (power in arbitrary units).

gives exemplary results for one of these bright points which are also valid for the others. Close inspection of the upper two diagrams reveals that the largest negative Doppler shift $(V)$ indicating the highest downward velocity precedes the second highest maximum of the minimumintensity $(I)$ fluctuation. While the whole line-forming region of $\mathrm{NaD}_{2}$ contributes to the $I$-signal, the $V$-signal is essentially determined by the velocity at the layer for which $\tau_{\text {line centre }} \approx 1$, marking the ultimate contact of the line-centre photons with the atmosphere. As the $V$-signal of the $\mathrm{NaD}_{2}$ line thus reflects the conditions in higher layers of the solar atmosphere than the $I$-signal, the findings from Fig. 8 may be interpreted by some perturbation propagating downward in the chromosphere. A caveat is appropriate here. In order to get deeper insight into the true atmospheric conditions, radiative transfer calculations are needed which consider the formation heights of intensity and velocity also in vertically inhomogeneous atmospheres. From the properties of the solar atmosphere studied at two different heights, Baudin et al. (1997) found intensity variations at the higher level preceding those in lower layers, yet it should be observed that their findings refer to the photosphere.

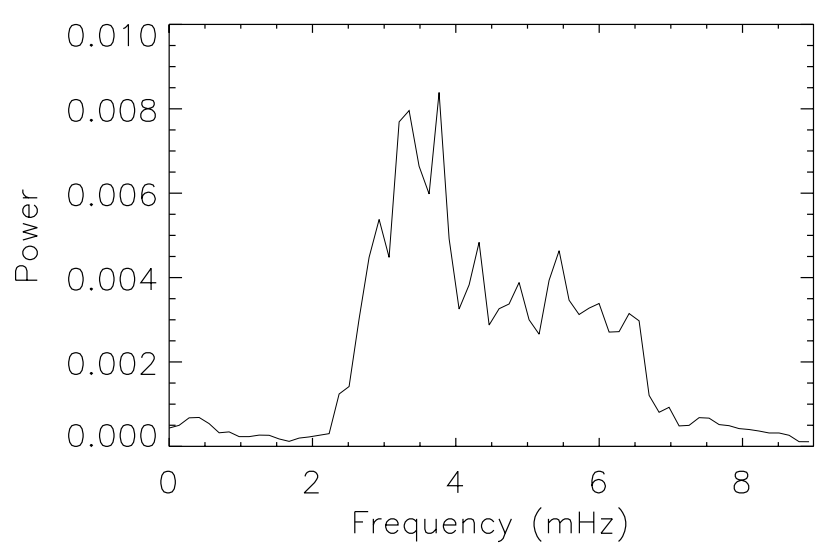

Fig. 7. Analogous to Fig. 6, the upper panel gives the Doppler shifts with time and the lower one the (smoothed) power spectrum for the same position in the field of view.

The power spectra in Fig. 8 reflect rather weak contributions from 3-min oscillations by comparatively low values around about $5.5 \mathrm{mHz}$ whereas both curves seem to bear a more pronounced signature of 5-min oscillations. The maximum peak in these power spectra occurs at $3.9 \mathrm{mHz}$ which corresponds to a period of about $4.3 \mathrm{~min}$.

The upper left diagram in Fig. 9 exhibits the power spectrum of the Doppler shift calculated over the time of conspicuously regular oscillations seen in Fig. 8. The other diagrams belong to the other bright points mentioned above also showing remarkably regular fluctuations of the Doppler shift. All power spectra display a prominent peak in a frequency range between about 3.5 and $4.5 \mathrm{mHz}$. The time spans of regular oscillations lasted from about 15 to $25 \mathrm{~min}$.

For additional information on the bright points, the corresponding small areas in the white-light images were inspected to see their photospheric counterparts. In all cases, they were identified with inconspicuous areas in intergranular lanes.

Espagnet et al. (1996) studied the spatial relation between the solar granulation and the 5-min oscillations of high-degree modes with apparent sizes of oscillating 

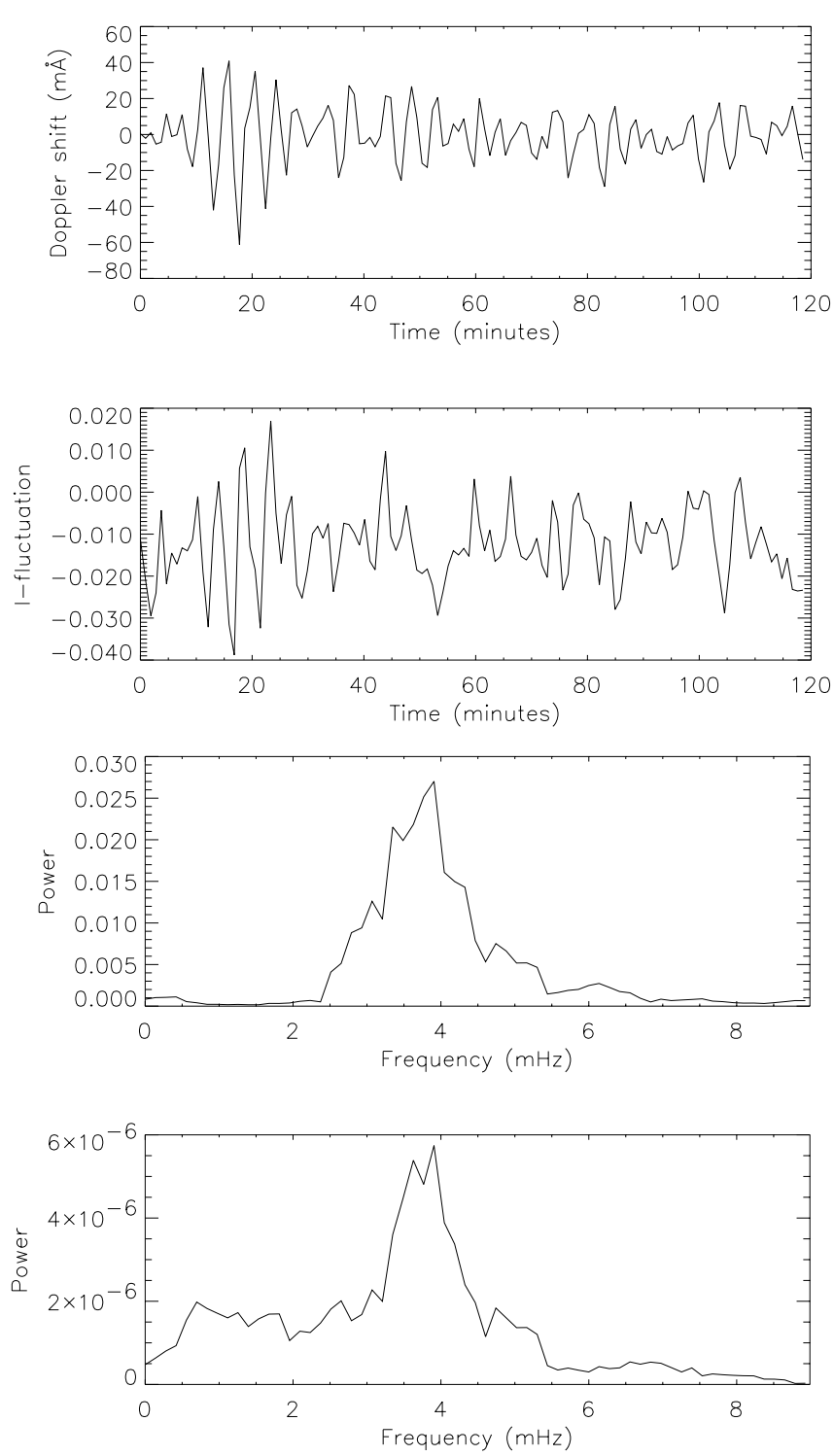

Fig. 8. Fluctuation of the Doppler shift and of the minimum intensity for a small intra-network area $\left(\left(1^{\prime \prime}\right)^{2}\right)$ exhibiting a bright point in one line-core image recorded over the whole time series; the corresponding (smoothed) power spectra of these fluctuations are shown in the lower two panels in the same order as the diagrams above.

cells of only few arcseconds. Their data suggest that the strongest oscillations are excited in intergranular spaces.

Goode et al. (1998) found strong seismic events to occur exclusively in dark intergranular lanes.

Considering the power spectra and the report on signatures of local wave excitation by intergranular lanes given by Hoekzema et al. (1998), one may assume some similar mechanism having left its marks here.

Yet another interpretation of the findings presented above is conceivable. Figure 8 clearly shows the preceding $V$-signal with respect to the $I$-signal over some time of quasi-regular oscillation. In order to quantify this relation, $V-I$ phase (and coherence) versus frequency have been calculated, yielding high coherence $(>0.8)$ for the relevant
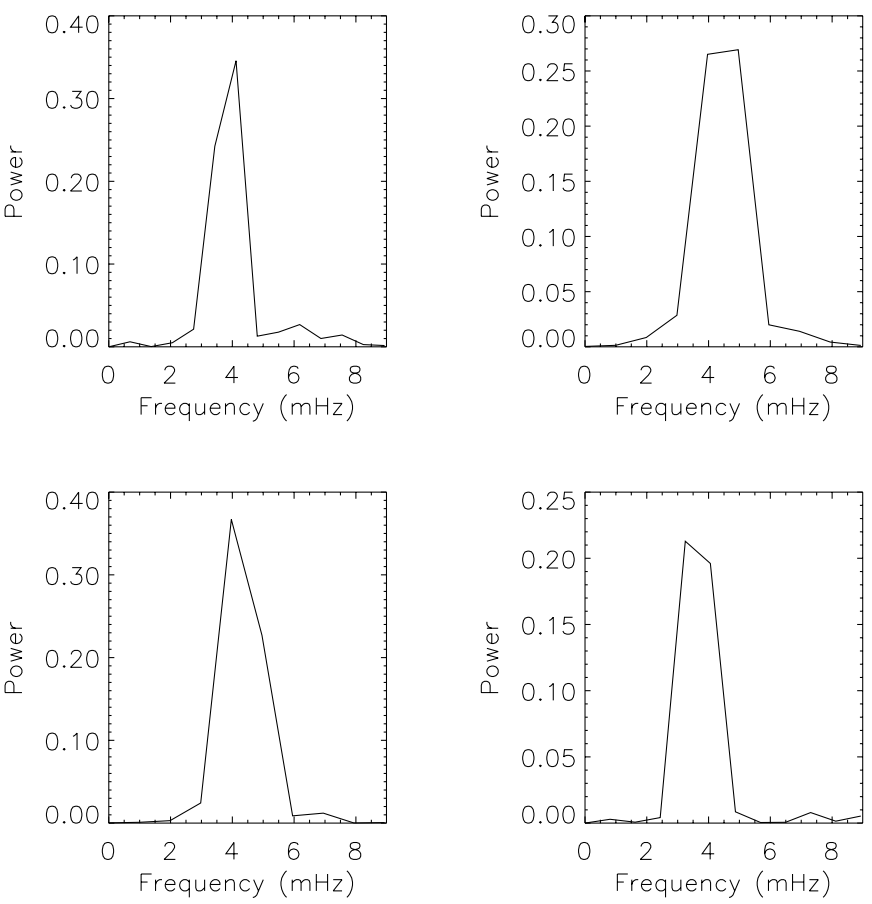

Fig. 9. Power spectra computed for the time spans of regularly oscillating Doppler shifts (from about 15 to $25 \mathrm{~min}$, corresponding to a frequency resolution of about 1 to $0.7 \mathrm{mHz}$ ) over small intra-network areas $\left(\left(1^{\prime \prime}\right)^{2}\right)$ exhibiting a bright point in at least one line-core image; the power spectrum in the upper left panel corresponds to the peculiar bright point whose behaviour has been illustrated in Fig. 8 .

frequencies and a $V-I$ phase relation between $-90^{\circ}$ and $-180^{\circ}$. More precisely, for the small feature belonging to Fig. 8 (and also to the upper left diagram of Fig. 9), a $V-I$ phase of about $-110^{\circ}$ was found. In the case of the upper right, the lower left, and the lower right diagram of Fig. 9 the corresponding $V-I$ phases yielded about $-95^{\circ}$, $-100^{\circ}$, and $-100^{\circ}$ (in the same order). For comparison, the $V-I$ phase values for the entire intra-network region within the field of view reach about $-85^{\circ}$ to $-90^{\circ}$ for the corresponding frequencies and thus suggest evanescent or standing waves. Irrespective of the size of the area (about $\left(1^{\prime \prime}\right)^{2}$ or the entire intra-network region), the $V-I$ phase values given here were obtained in the same way, namely by averaging the $V-I$ phase spectra of the corresponding individual pixels (for the whole time series).

However, the result for the small features under investigation can be indicative of evanescent waves (with increasing radiative damping) or of gravity waves. It cannot be decided here which interpretation is correct, but the latter remains plausible as a necessary precondition for the propagation of gravity waves is fulfilled: The BruntVäisälä frequency exceeds the frequencies for which most of the power is found in Fig. 9. Since it is only a little higher, the wavelength of the features under consideration is restricted to below $2 \mathrm{Mm}$, or $3^{\prime \prime}$. Kneer \& von Uexküll (1993) discussed the inference of gravity waves 
from their data showing a similar phase relation between the $I$-signals of $\mathrm{Mgb}_{2}$ and $\mathrm{CaK}$ (with $\mathrm{Ca}$ intensities leading $\mathrm{Mg}$ intensities at frequencies $\omega \leq 0.02 \mathrm{~s}^{-1}$ ). They presented circumstantial evidence of the detection of the signature of gravity waves as well.

\section{Conclusion and outlook}

The investigation of different types of bright points dealt with in a previous paper (Al et al. 1998b) has been continued, supporting and supplementing former results. Yet new questions arose:

- Are the persistent brightenings seen over a period of about 11 min actually connected with the magnetic field and is this the reason for their unusual longevity?

- Why are bright features that seem to be related to $\mathrm{K}$ grains less frequent when observed in the $\mathrm{Na}_{2}$ line rather than in CaII $\mathrm{K}_{2 \mathrm{~V}}$ ? What is the cause for the unusual 24-min oscillation of a small bright feature seen in the $\mathrm{NaD}_{2}$ line, but otherwise resembling a $\mathrm{K}$ grain in many respects?

- What makes the bright points seen in single line-core images oscillate so regularly over a time span between about 15 to $25 \mathrm{~min}$, yielding sharp maxima in the corresponding power spectra in a range from about 3.5 to $4.5 \mathrm{mHz}$ ? Do their $V-I$ phase spectra happen to reflect the manifestation of gravity waves?

The solar chromosphere does show a variety of features, some of which are barely understood because of their rare occurrence or because they are hard to detect. Each new known appearance thus adds to a better statistical background and helps to distinguish typical characteristics from incidental peculiarities. In order to increase the number of observed specimens, the optical set-up of the two-dimensional spectrometer in the VTT/Tenerife was changed in 1998, and a new time series was taken in the $\mathrm{Na}_{2}$ line. With a pixel resolution of 0.23 , still good enough for the detection of many small-scale features, the observed area was increased by about a factor of 2 . Moreover, the new time series consists of a larger number of scans and covers almost exactly 3 hours. This data set is expected to contain more unusual small chromospheric features awaiting revelation in the current data reduction process.

Furthermore, it would be helpful - yet very ambitious considering available post-focus instruments for solar observation - to study the chromosphere by two (or even more) such time series taken simultaneously in lines of different formation heights. For a reliable analysis, e.g. of the nature of waves to be discovered with such data sets, detailed non-LTE line formation modelling rather than simple height of formation estimates are highly desirable from the part of theory.

Acknowledgements. This work was partly supported by a scholarship from the University of Istanbul. The Gesellschaft für Technische Zusammenarbeit (GTZ) provided the computer equipment for further research results entered in this paper. The Vacuum Tower Telescope at the Spanish Observatorio del Teide of the Instituto de Astrofísica de Canarias/Tenerife is operated by the Kiepenheuer-Institut für Sonnenphysik in Freiburg, Germany.

\section{References}

Al, N. 1997, Thesis, University of Göttingen

Al, N., Bendlin, C., \& Kneer, F. 1998a, A\&A, 336, 743

Al, N., Bendlin, C., \& Kneer, F. 1998b, in JOSO Ann. Rep. 1998, ed. A. Antalová, H. Balthasar, \& A. Kučera, 133

Al, N., Bendlin, C., \& Kneer, F. 1999, A\&A, 342, 311

Baudin, F., Molowny-Horas, R., \& Koutchmy, S. 1997, A\&A, 326,842

Beckers, J. M. 1964, Thesis, University of Utrecht

Bendlin, C., \& Volkmer, R. 1995, A\&AS, 112, 371

Bendlin, C., Volkmer, R., \& Kneer, F. 1992, A\&A, 257, 817

Brandt, P. N., Rutten, R. J., Shine, R. A., \& Trujillo Bueno, J. 1992, in Cool Stars, Stellar Systems and the Sun, ed. M. S. Giampapa, \& J. A. Bookbinder, Proc. of the Seventh Cambridge Workshop, Astron. Soc. Pac. Conf. Ser., 26, 161

Brandt, P. N., Rutten, R. J., Shine, R. A., \& Trujillo Bueno, J. 1994, in Solar Surface Magnetism, ed. R. J. Rutten, \& C. J. Schrijver, NATO, ASI Ser. C433 (Kluwer Dordrecht), 251

Bray, R. J., \& Loughhead, R. E. 1974, The Solar Chromosphere (Chapman and Hall, London)

Cauzzi, G., Falchi, A., \& Falciani, R. 2000, A\&A, 357, 1093

Damé, L. 1985, in Theoretical Problems in High Resolution Solar Physics, ed. H. U. Schmidt, MPA 212 München, 244

Doyle, J. G., von den Oord, G. H. J., O'Shea, E., \& Banerjee, D. 1999, A\&A, 347, 335

Espagnet, O., Muller, R., Roudier, Th., et al. 1996, A\&A, 313, 297

Goode, Ph. R., Strous, L. H., Rimmele, Th. R., \& Stebbins, R. T. 1998, ApJ, 495, L27

Hoekzema, N. M., Rutten, R. J., Brandt, P. N., \& Shine, R. A. 1998, A\&A, 329, 276

Kneer, F., \& von Uexküll, M. 1993, A\&A, 274, 584

Kneer, F., \& von Uexküll, M. 1999, in Motions in the Solar Atmosphere, ed. A. Hanslmeier, \& M. Messerotti, 99

Lites, B. W., Rutten, R. J., \& Berger, T. E. 1999, ApJ, 517, 1013

Molowny-Horas, R. 1994, Thesis, University of Oslo

Narain, U., \& Ulmschneider, P. 1996, Space Sci. Rev., 75, 453

Nindos, A., \& Zirin, H. 1998, Sol. Phys., 179, 253

November, L. J., \& Simon, G. W. 1988, ApJ, 333, 427

Rutten, R. J., \& Uitenbroek, H. 1991, Sol. Phys., 134, 15

Sivaraman, K. R., Gupta, S. S., Livingston, W. C., et al. 2000, A\&A, 363, 279

Steffens, S., Hofmann, J., \& Deubner, F.-L. 1996, A\&A, 307, 288

Stellmacher, G., \& Wiehr, E. 1991, A\&A, 251, 675

Title, A. M., Topka, K. P., Tarbell, T. D., et al. 1992, ApJ, 393, 782

von Uexküll, M., \& Kneer, F. 1995, A\&A, 294, 252

Wang, H., Tang, F., Zirin, H., \& Wang, J. 1996, Sol. Phys., 165,223

Wellstein, S., Kneer, F., \& von Uexküll, M. 1998, A\&A, 335, 323

Yi, Z. 1992, Thesis, University of Oslo

Zhang, J., Lin, G., Wang, J., et al. 1998, Sol. Phys., 178, 245 